\title{
Maize Residue Interaction with High Quality Organic Materials: Effects on Decomposition and Nutrient Release Dynamics
}

\author{
Samuel T. Partey $\cdot$ Richard F. Preziosi • \\ Geoffrey D. Robson
}

Received: 20 October 2012 / Accepted: 29 January 2013 / Published online: 16 February 2013

(C) NAAS (National Academy of Agricultural Sciences) 2013

\begin{abstract}
The application of organic materials with wide carbon-to-nitrogen (C-to-N) ratios is known to cause initial immobilization of nutrients, unless $\mathrm{N}$ fertilizers are applied. In Sub-Saharan Africa, regular application of mineral fertilizers with organic residues is seldom practiced due to several socioeconomic constraints. In the present study, we assessed the biomass qualities of Zea mays, Tithonia diversifolia and Vicia faba and evaluated whether delayed decomposition and nutrient release of low quality residues will improve when mixed with high quality residues. Our hypothesis was that high quality organic residues have high $\mathrm{N}$ supply capabilities to improve decomposition and nutrient release of low quality materials when mixed together. Compared with $V$. faba and T. diversifolia biomasses, $Z$. mays residue was found to be relatively poor in quality as a result of its relatively low $\mathrm{N}$ concentration $(10.8 \mathrm{~g} / \mathrm{kg})$ and wider C-to- $\mathrm{N}$ ratio (37.2:1). The assessment on biomass quality was consistent with the results on decomposition. After one week, $42 \%$ of $Z$. mays residues had decomposed compared with more than $90 \%$ of $T$. diversifolia and $V$. faba residues. Further, the decomposition and $\mathrm{N}$ release rate of $Z$. mays tripled when mixed with either $T$. diversifolia or $V$. faba biomass. In this study, the initial $\mathrm{N}, \mathrm{C}$, lignin, C-to-N ratio, lignin-to-N ratio and (lignin + polyphenol)/ $\mathrm{N}$ ratio of the residues were useful indicators of degradability and nutrient release based on significant $(P<0.005)$ correlations. The study found that mixing $Z$. mays residue with either $T$. diversifolia or $V$. faba green biomass improved the $\mathrm{N}$ composition and C-to-N ratio of the mixture, which accounted for the improved decomposition and nutrient release rates of $Z$. mays residues in the mixture compared with sole $Z$. mays treatment. Our study therefore suggested that in places where inorganic fertilizers are limited, T. diversifolia and $V$. faba residues could be viable sources of $\mathrm{N}$ for improved decomposition and nutrient release of low quality residues.
\end{abstract}

Keywords Nutrient cycling $\cdot$ Residue chemistry $\cdot$ Biomass quality $\cdot$ Agroecosystems

\section{Introduction}

For many years, low-input agricultural technologies such as short duration planted tree fallows and green manuring with

S. T. Partey

Sustainable Consumption Institute, The University of

Manchester, 188 Waterloo Place, Oxford Road,

Manchester M13 9PL, UK

S. T. Partey $(\bowtie) \cdot$ R. F. Preziosi · G. D. Robson Faculty of Life Sciences, The University of Manchester, Michael Smith Building, Manchester M13 9PT, UK e-mail: sammtech147@yahoo.co.uk tree or crop residues have been demonstrated to offer a costeffective mechanism for increasing crop yields [17, 28, 36]. Despite experimental evidence that the application of organic materials improve overall soil fertility and crop yields [2, 16, 22], the selection of appropriate plant materials for soil fertility improvement and maintenance remains a major challenge in low-input agricultural systems [12]. Palm et al. [31] developed a decision support system for organic residue use and management in agroecosystems. According to this decision support system, high quality organic residues $(>2.5 \%$ nitrogen $(\mathrm{N}) ;<15 \%$ lignin; $<4 \%$ polyphenol) can be solely incorporated into soils with no $\mathrm{N}$ fertilizer additions, whist intermediate and less quality organic residues 
have been proposed to be applied in combination with $\mathrm{N}$ fertilizers $[6,31]$. The application of organic materials with wide carbon-to-nitrogen (C-to-N) ratios is known to cause initial immobilization of nutrients, which is overcome through the application of N fertilizers [3, 35]. In Sub-Saharan Africa, low and intermediate quality organic resources are more abundant in smallholder agriculture than high quality organic resources [13]. Like most parts of the tropics, maize (Zea mays) residues are among the most common intermediate quality organic resources in Sub-Saharan Africa, which although potential in soil management practices, are often, burnt before cropping. Although poor in quality due to relatively low $\mathrm{N}$ concentrations and wide $\mathrm{C}$-to$\mathrm{N}$ ratio, maize residues and $\mathrm{N}$ fertilizer combinations have shown tremendous positive influence on soil fertility and crop yields $[27,37,40]$. Whilst this practice remains a viable option for putting maize residues to judicious use in soil management practices, regular application of mineral fertilizers with organic residues are too seldom practiced $[23,24]$ in the region due to several socioeconomic constraints.

In the tropics, wide ranges of experiments and scholarly reports have confirmed that the nitrogen supply capabilities of the green manures of the Mexican sunflower (Tithonia diversifolia) and faba bean (Vicia faba) are comparable to that of inorganic fertilizers $[19,29,32]$. Contrary to maize residues, these high quality organic materials may be able to supply high amount of $\mathrm{N}$ at the early stages of crop growth, but for their short half-lives (in terms of decomposition and nutrient release) may only contribute a little to long-term maintenance of soil organic matter and soil fertility [14]. Furthermore, the harvesting of large quantities of high quality organic residues from adjacent cropping fields could have huge economic implications which could be minimized if the interaction between low quality crop residues and available high quality organic resources are found to be successful in cropping fields. In most smallholder agroecosystems where production is hugely dependent on organic matter in the soil, mixing organic materials of different qualities should be one viable soil management option to improve soil fertility and nutrient synchrony. The objective of our study was to assess the biomass qualities of Z. mays, V. faba and T. diversifolia and determine whether delayed decomposition and nutrient release of low quality residues will improve when mixed with high quality residues. Our hypothesis was that like inorganic $\mathrm{N}$ fertilizers, high quality organic residues have high $\mathrm{N}$ supply capabilities to improve decomposition and nutrient release of low quality materials.

\section{Materials and Methods}

Plant residue Characterization

Plant materials used in the study were the green biomasses of $V$. faba, T. diversifolia and Z. mays residues. Prior to the experiment, the aboveground portions of the plant materials were characterized for quality parameters. The plant materials were oven dried at $65^{\circ} \mathrm{C}$ till constant weight, grounded with a pestle and mortar and sieved to $0.5-\mathrm{mm}$ size. The sieved plant materials were analysed for total $\mathrm{N}$, $\mathrm{P}, \mathrm{K}, \mathrm{Ca}, \mathrm{Mg}, \mathrm{C}$, lignin and polyphenols in four replicates. The plant materials were either analysed solely or in a mixture (i.e. Z. mays $+T$. diversifolia; and Z. mays + $V$. faba in a $1: 1 \mathrm{w} / \mathrm{w}$ ratio). Nitrogen and $\mathrm{C}$ were determined simultaneously by dry combustion using LECO TruSpec $^{\text {TM }} \mathrm{CN}$ autoanalyzer (LECO Corporation). Total $\mathrm{K}, \mathrm{Ca}$ and $\mathrm{Mg}$ were determined by the dry ashing and atomic absorption spectrophotometry method as described by Eneji et al. [8] and Motsara and Roy [26]. Phosphorus was also determined in an ash solution by the ammonium phosphomolybdate method [26], whilst lignin was determined according to the acid detergent fiber method [39]. Polyphenols were determined by the method described by Gachengo et al. [10].

\section{Experimental Procedure, Design and Sampling}

The decomposition study was carried out using the aboveground portions of $V$. faba $(\mathrm{Vf}), T$. diversifolia $(\mathrm{Td})$ and Z. mays (M) either as sole or mixed treatments $(\mathrm{Vf}+\mathrm{M}$; and $\mathrm{Td}+\mathrm{M})$. The total aboveground biomasses of the plants equivalent to $20 \mathrm{~g}$ on a dry weight basis for both sole and mixed treatments were collected and placed in a $20 \times 20 \mathrm{~cm}$ rigid nylon litterbag of $1.5-\mathrm{mm}$ mesh size. For mixed treatments, plant residues were mixed in a $1: 1$ ratio $(w / w)$ [i.e. $\mathrm{Vf}(10 \mathrm{~g})+\mathrm{M}(10 \mathrm{~g})$ and $\mathrm{Td}(10 \mathrm{~g})+\mathrm{M}$ $(10 \mathrm{~g})]$. Litterbags were buried horizontally within $10 \mathrm{~cm}$ in trays filled with 2-mm sieved sandy-loam soil and arranged in a complete randomized design in five replications. Some properties of the soil used were $\mathrm{pH}$ (6.7), Total $\mathrm{N}(1.2 \mathrm{~g} / \mathrm{kg})$, organic $\mathrm{C}(13.8 \mathrm{~g} / \mathrm{kg})$, cation exchange capacity $\left(6.5 \mathrm{cmol}_{\mathrm{c}} / \mathrm{kg}\right)$, available $\mathrm{P}(2.4 \mathrm{mg} / \mathrm{kg})$, Clay $(5 \mathrm{~g} / \mathrm{kg})$, Sand $(604 \mathrm{~g} / \mathrm{kg})$ and Silt $(352 \mathrm{~g} / \mathrm{kg})$. The trays with litterbags were kept at $28{ }^{\circ} \mathrm{C}$ under glasshouse conditions. Moisture was kept at $55 \%$ of the dry weight of the soil and monitored using soil moisture metres ( 3 in 1 Hydroponic Soil Moisture Light PH Meter Analyzer). At one, two, four, eight and 12 weeks of decomposition, 
five litterbags (representing five replicates) per treatment were randomly selected from the trays to follow dry matter and nutrient losses. Plant materials remaining in the litterbags at each sampling time were separated from soil and organic debris by hand and oven dried at $65^{\circ} \mathrm{C}$ to constant weight. In order to correct for contamination by the mineral soil, samples were ashed at $500{ }^{\circ} \mathrm{C}$ for $4 \mathrm{~h}$. The difference between the dry weight of the decomposition materials and their ash contents was taken as the ash-free dry weight. Subsamples of the initial plant materials (at time 0) and plant materials remaining at each sampling time were analysed for nutrient concentrations by the analytical procedures described above.

\section{Mathematical Calculations and Statistical Analysis}

The amount of nutrients remaining in the litterbags at each sampling time was determined by multiplying the ash-free dry weight of the mass of plant material remaining by their nutrient concentrations. The percent of dry weight and nutrient remaining at each sampling time was calculated using the relation

$\mathrm{M}_{R}=\frac{M t}{M o} \times 100 \%$

where $M_{R}$ is the percent nutrient or quantity of plant material remaining, $M t$ is the amount of plant material or nutrient remaining at each sampling time and $M o$ is the initial weight of plant material or nutrient concentration.

The single three-parameter exponential model [43] was used to determine the decomposition and nutrient release rate constant $(k)$. The general form of the model was

$Y=D_{o}+D_{i} \mathrm{e}^{-k t}+$ error,

where $Y$ is the percent of initial material or nutrient remaining at sampling time $t, D_{o}$ is the recalcitrant pool fraction and $D_{i}$ is the difference $100-D_{o}$.

Half-life $\left(t_{1 / 2}\right)$, the time (in days) when $50 \%$ of plant materials had decomposed or $50 \%$ of nutrients had released, was calculated using the relation

$t_{\frac{1}{2}}=\frac{-\ln (2)}{k}$,

where $k$ is the decomposition or nutrient release rate obtained from Eq. 2.

Differences in data collected on the dry weight and nutrient remaining (on ash-free basis) among treatments were tested by means of Analysis of Variance (ANOVA) test. Where ANOVA test was significant, Tukey's post Hoc test (at $5 \%$ probability level) was used to determine where the differences exist among the means of the treatments. Percent dry weight and nutrient remaining (on ash-free basis) were regressed on time using nonlinear regression models. In addition, correlation and regression analysis were used to demonstrate the relationship between residue chemistry, decomposition and nutrient release rates. All statistical analyses were conducted using GENSTAT 11 [41].

\section{Results}

Chemical Characteristics of Plant Residues

Differences in chemical composition were apparent among treatments (Table 1). Nitrogen concentration was the highest in $V$. faba resulting in a significantly lowest C-to-N ratio $(P<0.05)$. Combining $Z$. mays residue to either $T$. diversifolia or $V$. faba residues altered their chemical compositions with $\mathrm{N}$ levels falling to about $43 \%$ in mixed $V$. faba $+Z$. mays relative to sole $V$. faba. Meanwhile, nitrogen levels in mixed treatments were more than twice that measured in sole $Z$. mays treatments. In addition, the high $\mathrm{N}$ concentration in mixed treatments resulted in a significantly low carbon-to-nitrogen ratio compared to sole Z. mays. T. diversifolia showed the greatest concentrations of $\mathrm{P}, \mathrm{Mg}$ and $\mathrm{K}$, whilst $V$. faba showed the greatest concentration of Ca. Lignin composition ranged from $41 \mathrm{~g} / \mathrm{kg}$ in $V$. faba to $58 \mathrm{~g} / \mathrm{kg}$ in $T$. diversifolia, whilst polyphenolic concentration increased in the order $Z$. mays $<V$. faba + $Z$. mays $<T$. diversifolia $+Z$. mays $<V$. faba $<$ T. diversifolia.

\section{Decomposition Dynamics}

The decomposition dynamics of all plant materials followed an exponential pattern with a significant $(P<0.001)$ nonlinear relationship between rate of decomposition and time (Table 2). ANOVA test confirmed significant $(P<0.001)$ effect of plant material type on decomposition rate $\left(k_{\mathrm{D}}\right.$ week $\left.^{-1}\right)$. Except in $Z$. mays, all plant materials recorded their highest dry weight losses during the first week of the experiment (Fig. 1). Percent dry weight remaining after the first week ranged from $9 \%$ in $V$. faba $+Z$. mays treatment to $58 \%$ in sole Z. mays treatment. Throughout the study period, $k_{\mathrm{D}}$ week $^{-1}$ was found to be significantly $(P<0.05)$ highest in $V$. faba $+Z$. mays (Table 3). Whilst all treatments showed a biphasic decomposition pattern, Z. mays generally showed a steady and uniform increase in decomposition through time. Relative to sole $V$. faba and sole Z. mays, decomposition rates increased significantly $(P<0.05)$ with the addition of the two $(V . f a b a+Z$. mays $)$ residues. In the first week alone, the $k_{\mathrm{D}}$ week $^{-1}$ of mixed $V$. faba $+Z$. mays was $26 \%$ and $77 \%$ higher than sole V. faba and sole Z. mays, respectively. Meanwhile, decomposition rates were 
Table 1 Chemical composition of sole and mixed organic residues used in the experiment

\begin{tabular}{|c|c|c|c|c|c|c|c|c|c|}
\hline \multirow[t]{2}{*}{ Treatment } & \multicolumn{8}{|c|}{ Chemical element $(\mathrm{g} / \mathrm{kg})$} & \multirow[t]{2}{*}{$\mathrm{C} / \mathrm{N}$} \\
\hline & $\mathrm{N}$ & $\mathrm{P}$ & $\mathrm{K}$ & $\mathrm{C}$ & $\mathrm{Ca}$ & $\mathrm{Mg}$ & Lig & Poly & \\
\hline $\mathrm{Td}$ & $28.1(0.8)^{\mathrm{bc}}$ & $5.2(0.2)^{\mathrm{c}}$ & $46.2(1.4)^{\mathrm{c}}$ & $400.6(4.2)^{\mathrm{a}}$ & $13.0(1.1)^{\mathrm{c}}$ & $8.3(0.2)^{\mathrm{c}}$ & $58.0(1.8)^{\mathrm{c}}$ & $18.0(0.7)^{\mathrm{d}}$ & $14.3(0.3)^{\mathrm{b}}$ \\
\hline Vf & $54.7(1.0)^{\mathrm{d}}$ & $2.5(0.2)^{\mathrm{a}}$ & $17.6(0.3)^{\mathrm{a}}$ & $427.4(2.4)^{\mathrm{cd}}$ & $27.0(1.1)^{\mathrm{e}}$ & $3.0(0.3)^{\mathrm{a}}$ & $41.0(1.4)^{\mathrm{a}}$ & $14.0(0.8)^{\mathrm{c}}$ & $7.8(0.2)^{\mathrm{a}}$ \\
\hline M & $10.8(0.6)^{\mathrm{a}}$ & $2.9(0.1)^{\mathrm{a}}$ & $20.6(0.7)^{\mathrm{a}}$ & $401.3(4.4)^{\mathrm{ab}}$ & $4.2(0.1)^{\mathrm{a}}$ & $2.9(0.1)^{\mathrm{a}}$ & $57.0(1.9)^{\mathrm{c}}$ & $5.6(0.3)^{\mathrm{a}}$ & $37.2(2.7)^{\mathrm{c}}$ \\
\hline $\mathrm{Td}+\mathrm{M}$ & $25.4(1.2)^{\mathrm{b}}$ & $4.3(0.1)^{\mathrm{b}}$ & $33.4(0.7)^{\mathrm{b}}$ & $417.6(5.1)^{\mathrm{bc}}$ & $8.2(0.6)^{\mathrm{b}}$ & $6.3(0.1)^{\mathrm{b}}$ & $56.7(1.3)^{\mathrm{c}}$ & $10.2(1.0)^{\mathrm{b}}$ & $17.8(0.8)^{\mathrm{b}}$ \\
\hline $\mathrm{Vf}+\mathrm{M}$ & $31.3(0.8)^{\mathrm{c}}$ & $2.7(0.2)^{\mathrm{a}}$ & $19.4(0.3)^{\mathrm{a}}$ & $436.2(1.5)^{\mathrm{d}}$ & $19.7(1.0)^{\mathrm{d}}$ & $2.8(0.2)^{\mathrm{a}}$ & $48.0(1.5)^{\mathrm{b}}$ & $8.1(0.4)^{\mathrm{ab}}$ & $13.9(0.4)^{\mathrm{b}}$ \\
\hline
\end{tabular}

Values are the means of four replicates. Values in parentheses are standard errors of means. Values with the same letters as superscript do not differ significantly according to Tukey test at $5 \%$ probability level

Lig lignin, Poly polyphenol, Td T. diversifolia, Vf V. faba, M Z. mays

Table 2 Nonlinear regression models for weight loss of plant materials

\begin{tabular}{llllrl}
\hline Treatment & Equation & SE & $R^{2}$ & $P$ value & $\begin{array}{l}\text { Half-life } \\
\text { (days) }\end{array}$ \\
\hline $\mathrm{Td}$ & $Y=3.91+95.50 \mathrm{e}^{-1.389 t}$ & 6.78 & 0.98 & 0.003 & 3.5 \\
$\mathrm{Vf}$ & $Y=6.12+93.77 \mathrm{e}^{-2.023 t}$ & 4.66 & 0.99 & $<0.001$ & 2.4 \\
$\mathrm{M}$ & $Y=6.17+92.83 \mathrm{e}^{-0.545 t}$ & 3.21 & 1.00 & $<0.001$ & 8.9 \\
$\mathrm{Td}+\mathrm{M}$ & $Y=8.82+90.95 \mathrm{e}^{-1.651 t}$ & 7.52 & 0.97 & 0.004 & 2.9 \\
$\mathrm{Vf}+\mathrm{M}$ & $Y=2.39+97.59 \mathrm{e}^{-2.616 t}$ & 2.63 & 1.00 & $<0.001$ & 1.9
\end{tabular}

Td T. diversifolia, Vf V. faba, $M$ Z. mays, SE standard error, $N=20$

comparable between sole $V$. faba and sole Z. mays after the fourth week. Between mixed T. diversifolia $+Z$. mays and sole Z. mays, significant $(P<0.05)$ differences in $k_{\mathrm{D}}$ week $^{-1}$ occurred only in the first week (Table 3 ). Contrary to the observations made between mixed $V$. faba + $Z$. mays and sole $V$. faba treatments, the addition of $Z$. mays to $T$. diversifolia reduced decomposition rates after the second week relative to sole $T$. diversifolia. However, $k_{\mathrm{D}}$ week $^{-1}$ was stabilized between sole $Z$. mays and mixed T. diversifolia $+Z$. mays after the first week. Generally, $k_{\mathrm{D}}$ week ${ }^{-1}$ as revealed by the predictive nonlinear models was higher in mixed treatments compared to their corresponding sole treatments (Table 2). The models also showed the half-life of sole $Z$. mays treatment to be less than one-third of that recorded for mixed Z. mays and either T. diversifolia or $V$. faba treatments.

\section{Nutrient Release Dynamics}

The nutrient release dynamics of all plant materials followed an exponential pattern (Fig. 2) with a significant $(P<0.05)$ nonlinear relationship between nutrient $(\mathrm{N}, \mathrm{P}$, $\mathrm{K}, \mathrm{Ca}, \mathrm{Mg}$ ) release rate and time (Table 4). ANOVA test also confirmed significant $(P<0.001)$ effect of plant material type on $\mathrm{N}, \mathrm{P}, \mathrm{K}, \mathrm{Ca}$ and $\mathrm{Mg}$ release rates (Table 5). The highest nitrogen loss in all plant materials occurred during the first week of decomposition with

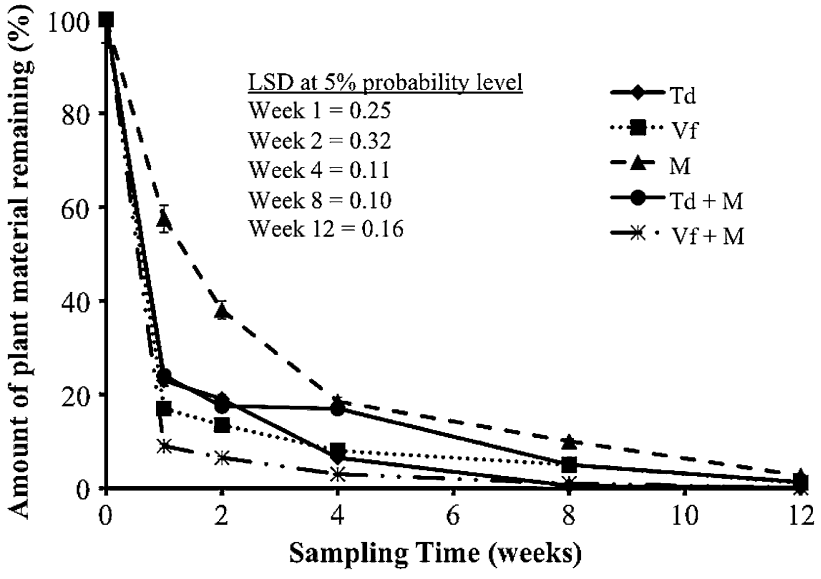

Fig. 1 Decomposition dynamics of sole and mixed organic residues over 12 weeks of placement in soil. Values are the means of five replicates. Error bars are the standard errors of means. LSD least significant difference, $T d T$. diversifolia, $V f V$. faba, $M$ Z. mays

nitrogen release rate $\left(k_{\mathrm{N}}\right.$ week $\left.{ }^{-1}\right)$ ranging from 0.88 in $Z$. mays to 3.17 in sole $V$. faba (Table 5). Nitrogen release rate in sole $Z$. mays was significantly lowest throughout the study period. Significant differences in $k_{\mathrm{N}}$ week $^{-1}$ between sole $V$. faba and mixed $V$. faba + Z. mays occurred at weeks 8 and 12 . Similarly, sole $T$. diversifolia and mixed $T$. diversifolia $+Z$. mays recorded comparable $k_{\mathrm{N}}$ week $^{-1}$ during the first four weeks of decomposition with significant $(P<0.05)$ differences occurring at weeks 8 and 12. As observed between sole V. faba and sole T. diversifolia treatments, comparable $k_{\mathrm{N}}$ week $^{-1}$ was recorded between mixed $V$. faba $+Z$. mays and mixed T. diversifolia $+Z$. mays treatments on the fourth week of the experiment. Except in sole Z. mays, nitrogen was the fast released nutrient in all plant materials (Table 6). Within each treatment, $\mathrm{N}$ loss constant was greater than mass loss.

Phosphorus release pattern was related to the decomposition and nitrogen release patterns of the plant materials. For most treatments, the highest phosphorus release rate $\left(k_{\mathrm{P}}\right.$ week $\left.^{-1}\right)$ occurred during the first week of the 
Table 3 Decomposition rates $\left(k_{\mathrm{D}} \%\right.$ week $\left.^{-1}\right)$ of sole and mixed species

\begin{tabular}{|c|c|c|c|c|c|}
\hline \multirow[t]{2}{*}{ Treatment } & \multicolumn{5}{|c|}{ Sampling period (week) } \\
\hline & 1 & 2 & 4 & 8 & 12 \\
\hline T. diversifolia & $1.470^{\mathrm{b}}$ & $0.830^{\mathrm{ab}}$ & $0.683^{\mathrm{b}}$ & $0.662^{\mathrm{b}}$ & $0.652^{\mathrm{b}}$ \\
\hline V. faba & $1.772^{\mathrm{b}}$ & $1.001^{\mathrm{b}}$ & $0.631^{\mathrm{b}}$ & $0.374^{\mathrm{a}}$ & $0.376^{\mathrm{a}}$ \\
\hline Z. mays & $0.553^{\mathrm{a}}$ & $0.484^{\mathrm{a}}$ & $0.422^{\mathrm{a}}$ & $0.288^{\mathrm{a}}$ & $0.301^{\mathrm{a}}$ \\
\hline T. diversifolia $+Z$. mays & $1.427^{\mathrm{b}}$ & $0.871^{\mathrm{ab}}$ & $0.443^{\mathrm{a}}$ & $0.374^{\mathrm{a}}$ & $0.362^{\mathrm{a}}$ \\
\hline V. faba + Z. mays & $2.408^{\mathrm{c}}$ & $1.498^{\mathrm{c}}$ & $0.877^{\mathrm{c}}$ & $0.863^{\mathrm{c}}$ & $0.989^{\mathrm{c}}$ \\
\hline SED & 0.121 & 0.154 & 0.052 & 0.049 & 0.077 \\
\hline
\end{tabular}

Values with the same letters as superscript do not differ significantly according to Tukey test at $5 \%$ probability level. $N=20$ $S E D$ standard errors of differences of means

Fig. $2 \mathrm{~N}, \mathrm{P}, \mathrm{K}, \mathrm{Ca}, \mathrm{Mg}$ and $\mathrm{C}$ release patterns in decomposing sole and mixed plant materials of T. diversifolia (Td), V. faba (Vf) and Z. mays (M) over 12 weeks of placement in soil. Data points are the means of five replicates. Error bars are the standard errors of means. $L S D$ least significant difference, $W k$ week
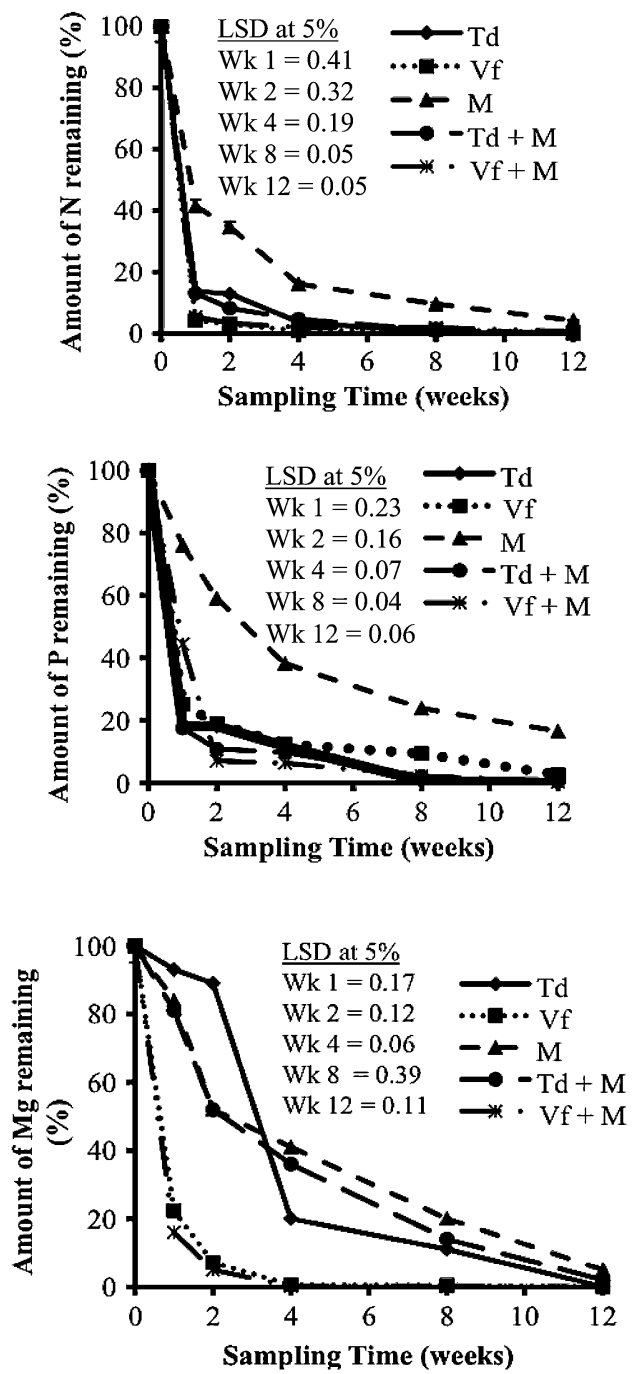
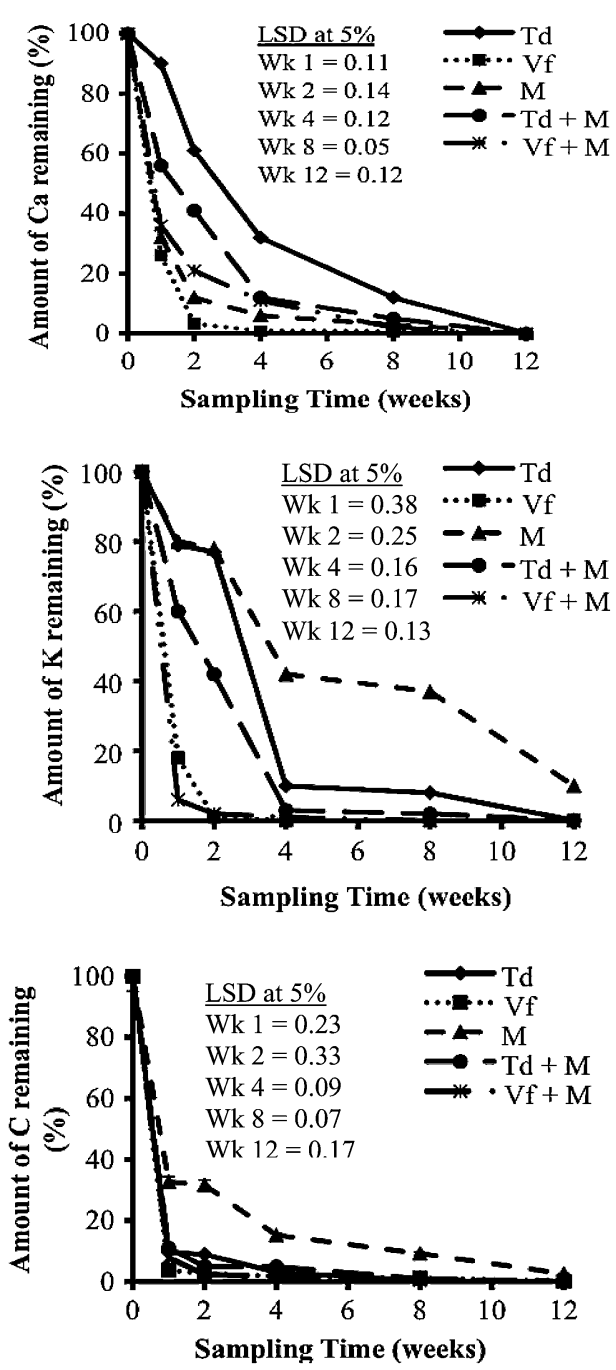

experiment. Phosphorus loss during the first week ranged from $24 \%$ in sole $Z$. mays to $82.5 \%$ in mixed $T$. diversifolia $+Z$. mays (Fig. 2). Meanwhile, the highest $k_{\mathrm{P}}$ week $^{-1}$ in mixed $V . f a b a+Z$. mays occurred on the 2 nd week of decomposition. Phosphorus release rate was significantly $(P<0.05)$ the lowest in sole $Z$. mays throughout the decomposition period. Between sole $T$. diversifolia and mixed $T$. diversifolia $+Z$. mays, significant differences in $k_{\mathrm{P}}$ week $^{-1}$ occurred on the $2 \mathrm{nd}, 8$ th and 12 th week of the experiment, whereas significant differences between sole $V$. faba and $V$. faba $+Z$. mays occurred throughout the experimental period. Meanwhile, the $k_{\mathrm{P}}$ week $^{-1}$ of sole 
Table 4 Nonlinear regression models for nutrient loss of plant materials

\begin{tabular}{llllll}
\hline $\begin{array}{l}\text { Nutrient/ Equation } \\
\text { treatments }\end{array}$ & SE & $R^{2}$ & $P$ value & $\begin{array}{l}\text { Half- } \\
\text { life } \\
\text { (days) }\end{array}$ \\
\hline
\end{tabular}

Nitrogen

$$
\begin{array}{lll}
\mathrm{Td} & Y=3.35+96.52 \mathrm{e}^{-2.052} \\
\mathrm{Vf} & Y=1.28+98.72 \mathrm{e}^{-3.487 t} \\
\mathrm{M} & Y=6.11+94.31 \mathrm{e}^{-0.565 t} \\
\mathrm{Td}+\mathrm{M} & Y=2.94+97.01 \mathrm{e}^{-2.169 t} \\
\mathrm{Vf}+\mathrm{M} & Y=1.89+98.1 \mathrm{e}^{-3.305 t}
\end{array}
$$

$\begin{array}{llll}5.42 & 0.99 & 0.001 & 2.4\end{array}$

$\begin{array}{llll}1.18 & 1.00 & <0.001 & 1.4\end{array}$

$$
\begin{array}{llll}
2.41 & 1.00<0.001 \quad 8.6
\end{array}
$$$$
3.23 \quad 1.00<0.001 \quad 2.2
$$$$
\begin{array}{llll}
3.05 & 1.00 & <0.001 & 1.5
\end{array}
$$

Phosphorus

$$
\begin{array}{ll}
\text { Td } & Y=6.27+93.47 \mathrm{e}^{-1.830 t} \\
\text { Vf } & Y=9.28+90.46 \mathrm{e}^{-1.598 t} \\
\mathrm{M} & Y=16.21+83.55 \mathrm{e}^{-0.332 t} \\
\mathrm{Td}+\mathrm{M} & Y=4.99+94.92 \mathrm{e}^{-1.938 t} \\
\mathrm{Vf}+\mathrm{M} & Y=0.96+100.02 \mathrm{e}^{-0.970 t}
\end{array}
$$

\begin{tabular}{|c|c|c|c|c|c|}
\hline $\mathrm{Td}$ & $Y=-6.60+112.10 \mathrm{e}^{-0.280 t}$ & 16.6 & 0.92 & 0.025 & 17.3 \\
\hline $\mathrm{Vf}$ & $Y=-0.42+100.48 \mathrm{e}^{-1.730 t}$ & 1.13 & 1.00 & $<0.001$ & 2.8 \\
\hline M & $Y=0.40+98.10 \mathrm{e}^{-0.162 t}$ & 9.49 & 0.95 & 0.010 & 30.0 \\
\hline $\mathrm{Td}+\mathrm{M}$ & $Y=-1.94+102.90 \mathrm{e}^{-0.502 t}$ & 6.49 & 0.98 & 0.002 & 9.7 \\
\hline $\mathrm{Vf}+\mathrm{M}$ & $Y=0.65+99.35 \mathrm{e}^{-2.90 t}$ & 0.83 & 1.00 & $<0.001$ & 1.7 \\
\hline \multicolumn{6}{|l|}{ Calcium } \\
\hline $\mathrm{Td}$ & $Y=89.3-2.12 \mathrm{e}^{-0.310 t}$ & 15.4 & 0.89 & 0.037 & 15.7 \\
\hline Vf & $Y=-0.10+100.26 \mathrm{e}^{-1.394 t}$ & 1.87 & 1.00 & $<0.001$ & 3.5 \\
\hline M & $Y=2.78+97.14 \mathrm{e}^{-1.188 t}$ & 2.01 & 1.00 & $<0.001$ & 4.1 \\
\hline $\mathrm{Td}+\mathrm{M}$ & $Y=1.29+97.69 \mathrm{e}^{-0.509 t}$ & 3.97 & 0.99 & $<0.001$ & 9.5 \\
\hline $\mathrm{Vf}+\mathrm{M}$ & $Y=3.88+95.30 \mathrm{e}^{-0.976 t}$ & 4.96 & 0.99 & 0.001 & 5.0 \\
\hline \multicolumn{6}{|l|}{ Magnesium } \\
\hline $\mathrm{Td}$ & $Y=-14.0+123.80 \mathrm{e}^{-0.203 \mathrm{t}}$ & 18.3 & 0.91 & 0.028 & 23.9 \\
\hline Vf & $Y=0.60+99.33 \mathrm{e}^{-1.488 t}$ & 0.96 & 1.00 & $<0.001$ & 3.3 \\
\hline M & $Y=3.89+96.44 \mathrm{e}^{-0.260 t}$ & 7.16 & 0.98 & 0.004 & 18.7 \\
\hline $\mathrm{Td}+\mathrm{M}$ & $Y=0.14+100.38^{-0.272 t}$ & 4.99 & 0.99 & 0.001 & 17.8 \\
\hline $\mathrm{Vf}+\mathrm{M}$ & $Y=0.52+99.43 \mathrm{e}^{-1.822 t}$ & 1.22 & 1.00 & $<0.001$ & 2.7 \\
\hline \multicolumn{6}{|l|}{ Carbon } \\
\hline $\mathrm{Td}$ & $Y=2.71+97.26 \mathrm{e}^{-2.526 t}$ & 3.87 & 0.99 & $<0.001$ & 1.9 \\
\hline Vf & $Y=1.40+98.60 \mathrm{e}^{-3.878 t}$ & 1.04 & 1.00 & $<0.001$ & 1.3 \\
\hline M & $Y=9.90+88.60 \mathrm{e}^{-1.047 t}$ & 9.29 & 0.96 & 0.008 & 4.6 \\
\hline $\mathrm{Td}+\mathrm{M}$ & $Y=2.47+97.51 \mathrm{e}^{-2.400 t}$ & 2.36 & 1.00 & $<0.001$ & 2.0 \\
\hline$V f+M$ & $Y=0.95+99.04 \mathrm{e}^{-2.652 t}$ & 0.87 & 1.00 & $<0.001$ & 1.8 \\
\hline
\end{tabular}$$
\begin{array}{llll}
8.15 & 0.97 & 0.005 & 2.6
\end{array}
$$$$
\begin{array}{llll}
5.62 & 0.99 & 0.002 & 3.0
\end{array}
$$$$
\begin{array}{llll}
1.29 & 1.00 & <0.001 \quad 14.6
\end{array}
$$$$
\begin{array}{llll}
4.75 & 0.99 & <0.001 \quad 2.5
\end{array}
$$$$
\begin{array}{llll}
6.14 & 0.99 & 0.002 & 5.0
\end{array}
$$

Potassium

Td T. diversifolia, $V f$ V. faba, $M$ Z. mays, $S E$ standard error, $R^{2}$ coefficient of multiple correlation, $P$ value significance of fit. $N=20$

\begin{tabular}{|c|c|c|c|c|c|}
\hline \multirow[t]{2}{*}{ Treatment } & \multicolumn{5}{|c|}{ Sampling period (weeks) } \\
\hline & 1 & 2 & 4 & 8 & 12 \\
\hline \multicolumn{6}{|l|}{ Nitrogen } \\
\hline T. diversifolia & $1.988^{\mathrm{b}}$ & $1.024^{\mathrm{b}}$ & $0.824^{\mathrm{bc}}$ & $0.726^{\mathrm{d}}$ & $0.710^{\mathrm{d}}$ \\
\hline V. faba & $3.170^{\mathrm{c}}$ & $1.753^{\mathrm{c}}$ & $1.089^{\mathrm{c}}$ & $0.589^{\mathrm{c}}$ & $0.576^{\mathrm{c}}$ \\
\hline Z. mays & $0.879^{\mathrm{a}}$ & $0.531^{\mathrm{a}}$ & $0.457^{\mathrm{a}}$ & $0.293^{\mathrm{a}}$ & $0.264^{\mathrm{a}}$ \\
\hline $\begin{array}{l}\text { T. diversifolia }+Z \text {. } \\
\text { mays }\end{array}$ & $2.033^{\mathrm{b}}$ & $1.251^{\mathrm{b}}$ & $0.770^{\mathrm{b}}$ & $0.576^{\mathrm{c}}$ & $0.576^{\mathrm{c}}$ \\
\hline$V \cdot f a b a+Z$. mays & $2.929^{\mathrm{c}}$ & $1.706^{\mathrm{c}}$ & $0.978^{\mathrm{bc}}$ & $0.495^{\mathrm{b}}$ & $0.426^{\mathrm{b}}$ \\
\hline SED & 0.196 & 0.151 & 0.089 & 0.023 & 0.024 \\
\hline \multicolumn{6}{|l|}{ Phosphorus } \\
\hline T. diversifolia & $1.707^{\mathrm{cd}}$ & $0.854^{\mathrm{b}}$ & $0.537^{\mathrm{b}}$ & $0.653^{\mathrm{d}}$ & $0.618^{\mathrm{d}}$ \\
\hline V. faba & $1.383^{\mathrm{c}}$ & $0.833^{\mathrm{b}}$ & $0.522^{\mathrm{b}}$ & $0.297^{\mathrm{b}}$ & $0.304^{\mathrm{b}}$ \\
\hline Z. mays & $0.274^{\mathrm{a}}$ & $0.264^{\mathrm{a}}$ & $0.241^{\mathrm{a}}$ & $0.179^{\mathrm{a}}$ & $0.150^{\mathrm{a}}$ \\
\hline $\begin{array}{l}\text { T. diversifolia }+Z \text {. } \\
\text { mays }\end{array}$ & $1.742^{\mathrm{d}}$ & $1.114^{\mathrm{c}}$ & $0.581^{\mathrm{b}}$ & $0.484^{\mathrm{c}}$ & $0.454^{\mathrm{c}}$ \\
\hline$V \cdot f a b a+Z$. mays & $0.812^{\mathrm{b}}$ & $1.327^{\mathrm{c}}$ & $0.690^{\mathrm{c}}$ & $0.483^{\mathrm{c}}$ & $0.499^{\mathrm{c}}$ \\
\hline SED & 0.112 & 0.075 & 0.031 & 0.020 & 0.027 \\
\hline \multicolumn{6}{|l|}{ Potassium } \\
\hline T. diversifolia & $0.236^{\mathrm{a}}$ & $0.131^{\mathrm{a}}$ & $0.576^{\mathrm{b}}$ & $0.316^{\mathrm{ab}}$ & $0.576^{\mathrm{b}}$ \\
\hline V. faba & $1.715^{\mathrm{b}}$ & $2.303^{\mathrm{b}}$ & $2.532^{\mathrm{e}}$ & $1.352^{\mathrm{c}}$ & $0.959^{\mathrm{c}}$ \\
\hline Z. mays & $0.223^{\mathrm{a}}$ & $0.124^{\mathrm{a}}$ & $0.217^{\mathrm{a}}$ & $0.124^{\mathrm{a}}$ & $0.192^{\mathrm{a}}$ \\
\hline $\begin{array}{l}\text { T. diversifolia }+Z \text {. } \\
\text { mays }\end{array}$ & $0.511^{\mathrm{a}}$ & $0.434^{\mathrm{a}}$ & $0.877^{\mathrm{c}}$ & $0.489^{\mathrm{b}}$ & $0.594^{\mathrm{b}}$ \\
\hline$V \cdot f a b a+Z$. mays & $2.813^{\mathrm{c}}$ & $1.956^{\mathrm{b}}$ & $1.151^{\mathrm{d}}$ & $1.439^{\mathrm{c}}$ & $1.151^{\mathrm{d}}$ \\
\hline SED & 0.180 & 0.120 & 0.053 & 0.081 & 0.064 \\
\hline \multicolumn{6}{|l|}{ Calcium } \\
\hline T. diversifolia & $0.105^{\mathrm{a}}$ & $0.099^{\mathrm{a}}$ & $0.079^{\mathrm{a}}$ & $0.062^{\mathrm{a}}$ & $0.518^{\mathrm{a}}$ \\
\hline V. faba & $1.343^{\mathrm{d}}$ & $1.706^{\mathrm{e}}$ & $1.197^{\mathrm{d}}$ & $0.622^{\mathrm{d}}$ & $0.548^{\mathrm{a}}$ \\
\hline Z. mays & $1.139^{\mathrm{c}}$ & $1.060^{\mathrm{d}}$ & $0.703^{\mathrm{c}}$ & $0.438^{\mathrm{bc}}$ & $0.484^{\mathrm{a}}$ \\
\hline $\begin{array}{l}\text { T. diversifolia }+Z \text {. } \\
\text { mays }\end{array}$ & $0.580^{\mathrm{b}}$ & $0.446^{\mathrm{b}}$ & $0.530^{\mathrm{b}}$ & $0.374^{\mathrm{b}}$ & $0.959^{\mathrm{b}}$ \\
\hline$V \cdot f a b a+Z$. mays & $1.022^{\mathrm{c}}$ & $0.780^{\mathrm{c}}$ & $0.552^{\mathrm{bc}}$ & $0.489^{\mathrm{c}}$ & $1.151^{\mathrm{c}}$ \\
\hline SED & 0.050 & 0.067 & 0.057 & 0.023 & 0.056 \\
\hline \multicolumn{6}{|l|}{ Magnesium } \\
\hline T. diversifolia & $0.073^{\mathrm{a}}$ & $0.058^{\mathrm{a}}$ & $0.402^{\mathrm{b}}$ & $0.276^{\mathrm{a}}$ & $0.576^{\mathrm{b}}$ \\
\hline V. faba & $1.496^{\mathrm{b}}$ & $1.323^{\mathrm{c}}$ & $1.271^{\mathrm{c}}$ & $0.670^{\mathrm{ab}}$ & $0.576^{\mathrm{b}}$ \\
\hline Z. mays & $0.174^{\mathrm{a}}$ & $0.327^{\mathrm{b}}$ & $0.223^{\mathrm{a}}$ & $0.201^{\mathrm{a}}$ & $0.250^{\mathrm{a}}$ \\
\hline $\begin{array}{l}\text { T. diversifolia }+Z \text {. } \\
\text { mays }\end{array}$ & $0.211^{\mathrm{a}}$ & $0.327^{\mathrm{b}}$ & $0.255^{\mathrm{a}}$ & $0.246^{\mathrm{a}}$ & $0.326^{\mathrm{a}}$ \\
\hline$V . f a b a+Z$. mays & $1.833^{\mathrm{c}}$ & $1.498^{\mathrm{d}}$ & $1.554^{\mathrm{d}}$ & $0.863^{\mathrm{b}}$ & $0.959^{c}$ \\
\hline SED & 0.083 & 0.056 & 0.030 & 0.185 & 0.053 \\
\hline
\end{tabular}

T. diversifolia and sole $V$. faba treatments were comparable until after the fourth week, the $k_{\mathrm{P}}$ week $^{-1}$ between the mixed treatments were significantly $(P<0.05)$ different at weeks 1 and 4. Furthermore, $P$ was the slowest released nutrient in sole $V$. faba and mixed $V$. faba + Z. mays treatments (Table 6).

The dynamic pattern of potassium during decomposition differed among treatments. Most treatments recorded their highest potassium release rates $\left(k_{\mathrm{K}}\right.$ week $\left.^{-1}\right)$ after the first
Table 5 Nutrient release rates $\left(\%\right.$ week $\left.^{-1}\right)$ of sole and mixed species

Values with the same letters as superscript do not differ significantly according to Tukey test at $5 \%$ probability level. $N=20$

$S E D$ standard errors of differences of means

two weeks of decomposition. During the first week, $k_{\mathrm{K}}$ week $^{-1}$ was significantly $(P<0.05)$ the greatest in $V$. faba + Z. mays with $94 \% \mathrm{~K}$ losses. During the same period, $\mathrm{K}$ retention was 79 and $80 \%$ in the decomposing 
Table 6 Order of nutrient release among decomposing plant materials over 12 weeks

\begin{tabular}{llllll}
\hline Treatments & \multicolumn{5}{l}{ Ranking order of release } \\
\cline { 2 - 6 } & 1 st & 2nd & $3 \mathrm{rd}$ & $4^{\text {th }}$ & 5 th \\
\hline T. diversifolia & $\mathrm{N}$ & $\mathrm{P}$ & $\mathrm{K}$ & $\mathrm{Ca}$ & $\mathrm{Mg}$ \\
V. faba & $\mathrm{N}$ & $\mathrm{K}$ & $\mathrm{Mg}$ & $\mathrm{Ca}$ & $\mathrm{P}$ \\
Z. mays & $\mathrm{Ca}$ & $\mathrm{N}$ & $\mathrm{P}$ & $\mathrm{Mg}$ & $\mathrm{K}$ \\
T. diversifolia + Z. mays & $\mathrm{N}$ & $\mathrm{P}$ & $\mathrm{Ca}$ & $\mathrm{K}$ & $\mathrm{Mg}$ \\
V. faba + Z. mays & $\mathrm{N}$ & $\mathrm{K}$ & $\mathrm{Mg}$ & $\mathrm{Ca}$ & $\mathrm{P}$ \\
\hline
\end{tabular}

Table 7 Linear correlation coefficients $(r)$ for the regression between initial chemistry of the plant materials and decomposition and nutrient release $(\mathrm{N}$ and $\mathrm{P})$ rates

\begin{tabular}{llll}
\hline $\begin{array}{l}\text { Chemical } \\
\text { composition }\end{array}$ & $\begin{array}{l}\text { Decomposition } \\
\text { rate }\end{array}$ & $\begin{array}{l}\text { Nitrogen } \\
\text { release rate }\end{array}$ & $\begin{array}{l}\text { Phosphorus } \\
\text { release rate }\end{array}$ \\
\hline $\mathrm{N}$ & $0.66^{* *}$ & $0.87 * * *$ & $\mathrm{~ns}$ \\
$\mathrm{P}$ & $\mathrm{ns}$ & $\mathrm{ns}$ & $0.83^{* * * *}$ \\
$\mathrm{~K}$ & $\mathrm{~ns}$ & $\mathrm{~ns}$ & $\mathrm{nd}$ \\
$\mathrm{C}$ & $0.83^{* * *}$ & $0.79 * * *$ & $\mathrm{~ns}$ \\
$\mathrm{Ca}$ & $\mathrm{nd}$ & $\mathrm{nd}$ & $\mathrm{ns}$ \\
$\mathrm{Mg}$ & $\mathrm{ns}$ & $\mathrm{ns}$ & $\mathrm{nd}$ \\
$\mathrm{Lignin}$ & $-0.61 * *$ & $-0.77 * * *$ & $\mathrm{~ns}$ \\
$\mathrm{Polyphenol}$ & $\mathrm{ns}$ & $\mathrm{ns}$ & $-0.56^{* *}$ \\
$\mathrm{C}: \mathrm{N}$ & $-0.79 * * *$ & $-0.88^{* * *}$ & $-0.47 *$ \\
$\mathrm{C}: \mathrm{P}$ & $\mathrm{ns}$ & $0.46^{*}$ & $-0.73^{* * *}$ \\
Lignin:N & $-0.83^{* * *}$ & $-0.91 * * *$ & $\mathrm{~ns}$ \\
(Lig + Poly):N & $-0.86^{* * *}$ & $-0.94 * * *$ & $\mathrm{~ns}$ \\
\hline
\end{tabular}

Lig lignin, Poly polyphenol, $n s$ not significant, *, ** and *** refer to significance at 5,1 and $0.1 \%$ probability levels respectively. $N=20$

matter of sole $T$. diversifolia and sole Z. mays, respectively. Compared with other treatments, the half-life of $\mathrm{K}$ in sole $Z$. mays was 30 days representing the slowest release nutrient in Z. mays. Between sole V. faba and V. faba+ $Z$. mays treatments, significant $(P<0.05)$ differences in $k_{\mathrm{K}}$ week $^{-1}$ occurred on weeks 1,4 and 12 , whereas $k_{\mathrm{K}}$ week $^{-1}$ in $T$. diversifolia + Z. mays and sole $T$. diversifolia were significantly $(P<0.05)$ different only on the $4^{\text {th }}$ week of decomposition. In addition, sole $Z$. mays and sole $T$. diversifolia recorded comparable $k_{\mathrm{K}}$ week ${ }^{-1}$ in most of the decomposition period. Between sole T. diversifolia and sole $V$. faba, significant differences in $k_{\mathrm{K}}$ week ${ }^{-1}$ occurred throughout the decomposition period. A similar observation was recorded between the mixed treatments ( $V . f a b a+$ $Z$. mays and T. diversifolia $+Z$. mays).

Among all the plant nutrients, $\mathrm{Ca}$ was the fastest release element in sole $Z$. mays. Unlike $\mathrm{N}$ and $\mathrm{P}$, there was high retention of $\mathrm{Ca}$ in the decomposing matter of most of the treatments even in the first week. Calcium losses during the first week of decomposition ranged from $10 \%$ in sole T. diversifolia to $68 \%$ in sole Z. mays. Generally, Ca release rate $\left(k_{\mathrm{Ca}}\right.$ week $\left.{ }^{-1}\right)$ as revealed by the nonlinear models was significantly $(P<0.05)$ the highest in sole $V$. faba and the lowest in sole T. diversifolia. Moreover, all plant materials showed slow release of $\mathrm{Mg}$ during the first week of decomposition except in sole $V$. faba and mixed $V$. faba $+Z$. mays treatments. Magnesium losses in the first week ranged from $84 \%$ in $V$. faba $+Z$. mays to only $7 \%$ in sole $T$. diversifolia treatments. Magnesium was the slowest released element among the nutrients investigated. Averagely, magnesium release rate $\left(k_{\mathrm{Mg}}\right.$ week $\left.^{-1}\right)$ was about one-third that of nitrogen. Meanwhile, $k_{\mathrm{Mg}}$ week $^{-1}$ was comparable between sole $Z$. mays and mixed $T$. diversifolia $+Z$. mays treatments. Furthermore, carbon mineralization pattern was closely related to the decomposition and $\mathrm{N}$ release patterns with the highest $\mathrm{C}$ losses occurring during the first week of the experiment. At the end of the first week, $\mathrm{C}$ losses ranged from approximately $66 \%$ in sole Z. mays to $97 \%$ in sole $V$. faba treatments. As revealed by the nonlinear models, $\mathrm{C}$ release rate $\left(k_{\mathrm{C}}\right.$ week $\left.^{-1}\right)$ followed the increasing order: Z. mays $<$ T. diversifolia $+Z$. mays $<T$. diversifolia $<V$. faba + $Z$. mays $<V$. faba. In addition, the results showed $k_{\mathrm{C}}$ week $^{-1}$ for all treatments greater than decomposition rates.

\section{Discussion}

Understanding the interactive effects of organic residues of differing chemistries on decomposition and nutrient release rates is important $[5,31,32]$ and provides significant insinuations for improving nutrient synchronization in organically managed agroecosystems. In the present study, we assessed the biomass qualities of $Z$. mays, T. diversifolia and $V$. faba and evaluated how the interaction between Z. mays and T. diversifolia or $V$. faba could influence their decomposition and nutrient release patterns. The assessment on biomass quality found $Z$. mays residues to be relatively poor in quality as a result of its relatively low $\mathrm{N}$ concentration and wider $\mathrm{C}-$ to- $\mathrm{N}$ ratio. Nitrogen and $\mathrm{C}$-to-N ratio values obtained in this study are not far from reports from previous investigations [33, 37, 40]. With regards to this chemical composition, applying $Z$. mays residues as an organic fertilizer could potentially result in net $\mathrm{N}$ immobilization unless $\mathrm{N}$ fertilizers are added [5]. Meanwhile, the levels of $\mathrm{N}$ and C-to-N in $V$. faba and T. diversifolia were found to be high and within recommended levels for organic fertilization as reported by Palm et al. [31]. Whilst the biochemical characteristics of plant materials can differ considerably, depending on plant species, sources of nitrogen applied, stage of plant growth and edaphic and climatic conditions [44], the high $\mathrm{N}$ and low C-to-N ratio in $T$. diversifolia and $V$. faba are similarly reported $[10,18,32]$. Although mixed residues had equal 
proportions of separate components, their chemical concentrations were hugely altered and not typical of what could be potentially predicted in theory as described by Gartner and Cardon [11]. Meanwhile, N levels were comparatively lower in mixed residues than sole $T$. diversifolia and sole $V$. faba treatments although within recommended levels for green manuring [31]. The reduced $\mathrm{N}$ levels in mixed treatments can be attributed to the presence of the $Z$. mays residues with relatively low $\mathrm{N}$ concentration.

The results of our study demonstrated significant differences in decomposition and nutrient release rates between sole and mixed organic residues. Although some authors have contested about the applicability of using rates constants obtained from the single exponential model in describing best fitted decomposition and nutrient release patterns of plant materials $[9,43]$, the high $R^{2}$ values obtained from this study makes the single exponential model seem applicable. Among treatments, statistical comparisons revealed significantly $(P<0.05)$ the greatest decomposition rate in $V$. faba $+Z$. mays treatments. With the exception of sole $Z$. mays, all treatments recorded halflives within a week. Whilst similar studies are rare for comparison, rapid decomposition and nutrient release patterns of $T$. diversifolia are similarly reported $[10,18,32]$. Comparatively, the decomposition rates of sole $V$. faba and sole $Z$. mays were considerably lower than mixed $V$. faba $+Z$. mays. In the first week alone, the decomposition rate of $V$. faba $+Z$. mays was four times higher than sole $Z$. mays treatments. Assuming that the decomposition rate of a single plant residue in a mixture with other residues is a fraction of the decomposition rate of the total mixture [11] then the observations supports the idea that adding $Z$. mays residue to $V$. faba could substantially improve decomposition rates of $Z$. mays. With high decomposition rates also recorded in mixed $T$. diversifolia $+Z$. mays treatments, the study revealed that adding $Z$. mays to $T$. diversifolia could improve the decomposition rate of $Z$. mays three times higher than that recorded for sole Z. mays. The results on decomposition were also consistent with the nutrient release patterns observed. Except in the case of $\mathrm{Ca}$, the results of the study confirmed that mixing $V$. faba or $T$. diversifolia with $Z$. mays could substantially alleviate delayed nutrient release rates in $Z$. mays (Table 6). Although investigations in decomposer abundance and community composition were not covered in our research, we in part attribute the significant differences in decomposition and nutrient release patterns between the sole and mixed plant residues to the development of different decomposer communities on plant materials based on their intrinsic properties [7]. Recent and earlier investigations have confirmed that mixing leaves from species with varying chemical composition and qualities changes the chemical environment and physically alters the total litter surface where decomposition is occurring $[11,20]$. These alterations are noted to affect decomposer abundance and activity [11, 15, 42], which can significantly vary the decomposition and nutrient release patterns of sole and mixed plant residues. Among the cations $(\mathrm{K}, \mathrm{Ca}$ and $\mathrm{Mg}$ ), potassium recorded the fastest release rate. Potassium release rate was found to be inconsistent with the results on decomposition, which supports the assertion by Bhupinderpal-Singh and Rengel [3] that leaching is the primary process influencing $\mathrm{K}$ losses. According to BhupinderpalSingh and Rengel [3], potassium is not present in plant tissues in organic structures; therefore, its release from residues is not dependent on residue decomposition. Furthermore, the study confirmed that the decomposition and nutrient release rates of the plant materials were dependent on their chemistry (Table 7). The role of chemical composition in organic matter decomposition and nutrient release is well documented $[1,5,21,38]$ and has been shown to be more influential than climatic factors $[25,30]$. In this study, initial $\mathrm{N}, \mathrm{C}$, lignin, $\mathrm{C}$-to-N ratio, lignin-to- $\mathrm{N}$ ratio and (lignin + polyphenol) $/ \mathrm{N}$ ratio were useful indicators in predicting the degradability of the organic materials investigated (Table 7). In addition, the study found significant relationships between $k_{\mathrm{N}}$ week ${ }^{-1}$ and initial N $(r=0.87, P<0.01)$, C $(r=0.79, P<0.001)$, lignin $(r=-0.77, \quad P<0.001), \quad$ C-to-N ratio $(r=-0.88$, $P<0.001)$, C-to-P ratio $(r=0.46, P<0.05)$, lignin-to-N ratio $(r=-0.91, P<0.001)$ and (lignin + polyphenol) $/ \mathrm{N}$ ratio $(r=-0.94, P<0.001)$. These significant correlations are consistent with the reports of several authors $[3,4$, $34]$ and provide relevant insinuations for organic resource management in tropical agroecosystems.

\section{Conclusions}

For the production systems that hugely depend on organic matter, the results of this study provide significant information for organic residue use and management. Compared with $V$. faba and $T$. diversifolia biomasses, the study found $Z$. mays residue to be of low quality based on its chemical composition and relatively low decomposition and nutrient release rates. The biomass quality of $Z$. mays provided a reasonable premise to assume that the application of Z. mays residues on soils could induce $\mathrm{N}$ immobilization unless applied together with $\mathrm{N}$ fertilizers. Given that resource poor farmers have huge challenges meeting the fertilizer requirements on farmlands, we conducted our decomposition study on the hypothesis that like $\mathrm{N}$ fertilizers, the $\mathrm{N}$ supply capabilities of high quality organic resources (in this case, $V$. faba and T. diversifolia residues) could enable them improve the decomposition and nutrient release rates of $Z$. mays when mixed together. It was 
evident from the results that the decomposition and nutrient release of low organic residues will significantly improve when mixed with high quality organic residues. In this study, the decomposition and $\mathrm{N}$ release rate of $Z$. mays tripled when mixed with either $T$. diversifolia or $V$. faba biomass. The results showed that mixing $Z$. mays residue with either $T$. diversifolia or $V$. faba green biomass improved the $\mathrm{N}$ composition and $\mathrm{C}$-to-N ratio of the mixture, which accounted for the improved decomposition and nutrient release rates of $Z$. mays residues in the mixture compared with sole $Z$. mays treatment. We therefore suggested that in places where inorganic fertilizers are limited, T. diversifolia and $V$. faba residues could be viable sources of $\mathrm{N}$ for improved decomposition and nutrient release of low quality residues. Whilst the results of our study confirmed improved decomposition and nutrient release rates of a low quality organic residue when mixed with a high quality residue, it was limited in demonstrating how much of the nutrients released could be available in soil. The study was also limited in demonstrating the implications of accelerated decomposition on long-term build up of soil fertility. Further studies could therefore tackle the effects of organic residue quality and $\mathrm{N}$ release patterns on soil fertility indicators and agronomic efficiency.

Acknowledgments The authors wish to express their sincere gratitude to the Sustainable Consumption Institute, The University of Manchester for providing funding for Samuel Partey.

\section{References}

1. Abarchi I, Zhan-yu Z, Vanlauwe B, Xiang-ping G, Wei-mu W, Ong'or BTI, Timbely D (2009) Effects of plant age and rock phosphate on quality and nutrient release of legume residue. Pedosphere 19:78-85

2. Beedy TL, Snapp SS, Akinnifesi FK, Sileshi GW (2010) Impact of Gliricidia sepium intercropping on soil organic matter fractions in a maize-based cropping system. Agric Ecosyst Environ 138:139-146

3. Bhupinderpal-Singh, Rengel Z (2007) The role of crop residues in improving soil fertility. In: Marschner P, Rengel Z (eds) Nutrient cycling in terrestrial ecosystems, Soil Biology, Vol 10. Springer, Berlin, Heidelberg pp 183-214

4. Bhupinderpal-Singh, Rengel Z, Bowden JW (2006) Carbon, nitrogen and sulphur cycling following incorporation of canola residue of different sizes into a nutrient-poor sandy soil. Soil Biol Biochem 38:1591-1597

5. Brady NC, Weil RR (2004) Elements of the nature and properties of soils, 2nd edn. Prentice Hall, Upper Saddle River

6. Chivenge P, Vanlauwe B, Gentile R, Six J (2011) Organic resource quality influences short-term aggregate dynamics soil organic carbon and nitrogen accumulation. Soil Biol Biochem 43:657-666

7. Cobo JG, Barrios E, Kass DCL, Thomas RJ (2002) Decomposition and nutrient release by green manures in a tropical hillside agroecosystem. Plant Soil 240:331-342
8. Eneji AE, Yamamoto S, Wen G, Inanaga S, Honna T (2005) Comparative evaluation of wet digestion and dry ashing methods for the determination of some major and minor nutrients in composted manure. Toxicol Environ Chem 87:147-158

9. Ezcurra E, Becerra J (1987) Experimental decomposition of litter from the Tamaulipan cloud forest: a comparison of four simple models. Biotropica 19:290-296

10. Gachengo CN, Palm CA, Jama B, Othieno C (1999) Tithonia and Senna green manures and inorganic fertilizers as phosphorus sources for maize in western Kenya. Agrofor Syst 44:21-36

11. Gartner TB, Cardon ZG (2004) Decomposition dynamics in mixed-species leaf litter. Oikos 104:230-246

12. George TS, Gregory PJ, Robinson JS, Buresh RJ, Jama B (2002) Utilization of soil organic phosphorus by agroforestry and crop species in the field in western Kenya. Plant Soil 246:53-63

13. Giller KE, Cadisch G, Mugwira LM (1998) Potential benefits from interactions between mineral and organic nutrient sources. In: Waddington SR (ed) Soil fertility research for maize-based farming systems in Malawi and Zimbabwe. Soil Fertility Network and CIMMYT-Zimbabwe, Harare, pp 155-158

14. Handayanto E, Giller KE, Cadisch G (1997) Regulating N release from legume tree prunings by mixing residues of different quality. Soil Biol Biochem 29:1417-1426

15. Hansen RA (1999) Red oak litter promotes a microarthropod functional group that accelerates its decomposition. Plant Soil 209:37-45

16. Ikerra ST, Semu E, Mrema JP (2007) Combining Tithonia diversifolia and Minjingu phosphate rock for improvement of $\mathrm{P}$ availability and maize grain yields on a chromic acrisol in Morogoro, Tanzania. In: Bation A, Waswa B, Kihara J, Kimetu J (eds) Advances in integrated soil fertility management in SubSaharan Africa: challenges and opportunities. Springer, Dordrecht, pp 333-344

17. Jama B, Kiwia A (2009) Agronomic and financial benefits of phosphorus and nitrogen sources in western Kenya. Exp Agric 45:241-260

18. Jama B, Palm CA, Buresh RJ, Niang AI, Gachengo C, Nziguheba G, Amadalo B (2000) Tithonia diversifolia as a green manure for soil fertility improvement in western Kenya: a review. Agrofor Syst 49:201-221

19. Jensen ES, Peoples MB, Hauggaard-Nielsen H (2010) Faba bean in cropping systems. Field Crop Res 115:203-216

20. Kaneko N, Salamanca EF (1999) Mixed leaf litter effects on decomposition rates and soil microarthropod communities in an oak-pine stand in Japan. Ecol Res 14:131-138

21. Lupwayi NZ, Clayton GW, O'Donovan JT, Harker KN, Turkington TK, Soon YK (2007) Phosphorus release during decomposition of crop residues under conventional and zero tillage. Soil Tillage Res 95:231-239

22. Makumba W, Akinnifesi F, Janssen B, Oenema O (2006) The long-term impact of a gliricidia-maize intercropping system on carbon sequestration in southern Malawi. Agric Ecosyst Environ 118:237-243

23. Mateete B, Nteranya S, Paul WL (2010) Restoring soil fertility in Sub-Sahara Africa. Adv Agron 108:183-236

24. Mazur R, Onzere S (2009) Social networks and status in adopting agricultural technologies and practices among small-scale farmers in Uganda. In: Sanginga PC, Waters-Bayers A, Kaaria S, Njuki J, Wettasinha $C$ (eds) Innovation Africa: enriching farmers livelihoods. Earthscan, London, pp 120-134

25. Meentemeyer V (1978) Macroclimate and lignin control of litter decomposition rates. Ecology 59:465-472

26. Motsara MR, Roy RN (2008) Guide to laboratory establishment for plant nutrient analysis. FAO fertilizer and plant nutrition bulletin. Food and Agriculture Organization, Rome, p 219 
27. Mucheru-Muna M, Mugendi D, Kung'u J, Mugwe J, Bationo A (2007) Effects of organic and mineral fertilizer inputs on maize yield and soil chemical properties in a maize cropping system in Meru South District, Kenya. Agrofor Syst 69:189-197

28. Niang A, Gathumbi S, Amadalo B (1996) The potential of short duration improved fallow for crop productivity enhancement in the highlands of western Kenya. E Afr Agric For J 62:103-114

29. Nziguheba G, Merckx R, Palm CA, Rao MR (2000) Organic residues affect phosphorus availability and maize yields in a Nitisol of western Kenya. Biol Fertil Soils 32:328-339

30. Palm CA, Sanchez PA (1990) Decomposition and nutrient release patterns of the leaves of three tropical legumes. Biotropica 22:330-338

31. Palm CA, Gachengo CN, Delve RJ, Cadisch G, Giller K (2001) Organic inputs for soil fertility management in tropical agroecosystems: application of an organic resource database. Agric Ecosyst Environ 83:27-42

32. Partey ST, Quashie-Sam SJ, Thevathasan NV, Gordon AM (2011) Decomposition and nutrient release patterns of the leaf biomass of the wild sunflower (Tithonia diversifolia): a comparative study with four leguminous agroforestry species. Agrofor Syst 8:123-134

33. Sakala WD, Cadisch G, Giller KE (2000) Interactions between residues of maize and pigeonpea and mineral $\mathrm{N}$ fertilizers during decomposition and $\mathrm{N}$ mineralization. Soil Biol Biochem 32:679-688

34. Salas AM, Elliott ET, Westfall DG, Cole CV, Six J (2003) The role of particulate organic matter in phosphorus cycling. Soil Sci Soc Am J 67:181-189

35. Sanchez PA (2002) Soil fertility and hunger in Africa. Science 295:2019-2020
36. Sanchez PA, Shepherd KD, Soule MJ, Place FM, Buresh RJ, Izac AN (1997) Soil fertility replenishment in Africa: an investment in natural resource capital. In: Buresh RJ, Sanchez PA, Calhoun F (eds) Replenishing soil fertility in Africa: SSSA special publication number 51. ASA and SSSA, Madison, pp 1-46

37. Smaling EMA, Stoorvogel JJ, de Jager A (2002) Decision making on integrated nutrient management through the eyes of the scientist, the land-user and the policy maker. In: Vanlauwe B, Diels J, Sanginga N, Merckx R (eds) Integrated plant nutrient management in Sub-Saharan Africa. CAB International, Wallingford, pp 265-283

38. Troeh FR, Thompson LM (2005) Soils and soil fertility, 6th edn. Blackwell, Ames, p 489

39. van Soest PJ (1963) Use of detergents in the analysis of fibrous feeds. II. A rapid method for the determination of fibre and lignin. J Assoc Agric Chem 46:829-835

40. Vanlauwe B, Aihou K, Aman S, Iwuafor ENO, Tossah BK, Diels J, Sanginga N, Lyasse O, Merckx R, Deckers J (2001) Maize yield as affected by organic inputs and urea in the West African moist savannah. Agron J 93:1191-1199

41. VSN International Ltd (2008) GENSTAT for Windows, 11th edn. VSN International, Hertfordshire

42. Wardle DA (2002) Communities and ecosystems: linking the aboveground and belowground components. Princeton University Press, Princeton

43. Wieder R, Lang G (1982) A critique of the analytical methods used in examining decomposition in litterbags. Ecology 63:1636-1642

44. Xu JM, Tang C, Chen ZL (2006) Chemical composition controls residue decomposition in soils differing in initial $\mathrm{pH}$. Soil Biol Biochem 38:544-552 